\title{
Meta
}

Journal des traducteurs

Translators' Journal

\section{Mon ami m’a dit}

\section{Henri Awaiss}

Volume 50, numéro 1, mars 2005

Enseignement de la traduction dans le monde

Teaching Translation Throughout the World

URI : https://id.erudit.org/iderudit/010657ar

DOI : https://doi.org/10.7202/010657ar

Aller au sommaire du numéro

Éditeur(s)

Les Presses de l'Université de Montréal

ISSN

0026-0452 (imprimé)

1492-1421 (numérique)

Découvrir la revue

\section{Citer cet article}

Awaiss, H. (2005). Mon ami m'a dit. Meta, 50(1), 60-66.

https://doi.org/10.7202/010657ar

\section{Résumé de l'article}

Un milieu bilingue ou plurilingue serait-il l'espace idéal pour enseigner/apprendre la traduction et l'interprétation ? Les gens de ce paradis rêvé seraient-ils comme prédestinés à ce métier ? Une formation académique serait-elle un non-sens ? L'ÉTIB, de par son expérience, cherche non seulement à rendre compte d'une situation originale mais à contribuer à une réflexion traductologique vécue au quotidien.
Ce document est protégé par la loi sur le droit d'auteur. L’utilisation des services d’Érudit (y compris la reproduction) est assujettie à sa politique d'utilisation que vous pouvez consulter en ligne.

https://apropos.erudit.org/fr/usagers/politique-dutilisation/ 


\title{
Mon ami m'a dit
}

\author{
HENRI AWAISS \\ Université Saint-Joseph, Beyrouth, Liban \\ hawais@usj.edu.lb
}

\begin{abstract}
RÉSUMÉ
Un milieu bilingue ou plurilingue serait-il l'espace idéal pour enseigner/apprendre la traduction et l'interprétation? Les gens de ce paradis rêvé seraient-ils comme prédestinés à ce métier? Une formation académique serait-elle un non-sens ? L'ÉTIB, de par son expérience, cherche non seulement à rendre compte d'une situation originale mais à contribuer à une réflexion traductologique vécue au quotidien.
\end{abstract}

\begin{abstract}
Is the milieu of bilingualism or multilingualism the ideal setting to learn / teach translation or interpretation? Are people predestined in this world to practice this profession? Is academic preparation for it illogical? ETIB not only wishes to transmit this unique situation, but also to contribute to a traductologic reflection in daily practice.
\end{abstract}

\section{MOTS-CLÉS/KEYWORDS}

Liban, enseignement, profession, milieu bilingue

En visite à Beyrouth pour la première fois, mon ami, professeur invité ${ }^{1}$, m'a raconté dès sa descente de l'avion qu'il était assis à côté d'un Canadien d'origine libanaise. La conversation entre les deux passagers s'est déroulée en français et en anglais sur fond arabo-oriental: Schéhérazade présente parmi eux inventait à son roi mille et un rêves et transformait l'avion en un tapis volant. Quant aux poètes, écrivains et hommes de lettres arabes français et anglais cités tout au long des quatre heures du vol, ils s'installèrent confortablement dans les sièges de cet airbus reliant Paris à Beyrouth.

Le deuxième jour, dans le taxi qui l'amenait à l'université, mon ami a eu droit - non sans surprise et étonnement - à un récit historique, en français, par son chauffeur sur le passage du général de Gaulle dans la ville; c'était, me disait-il, un témoignage vivant dans lequel son interlocuteur s'efforçait d'expliquer son attachement ainsi que son respect à une deuxième, voire troisième langue, l'arabe étant bien sûr la première ${ }^{2}$.

Quelques jours plus tard, le soir à table devant un magnifique mezzé ${ }^{3}$ aux multiples petites assiettes merveilleusement assorties à l'ombre de quelques bouteilles d'arak et de vin, mon ami était agréablement surpris par la diversité des goûts, le métissage des senteurs et le mélange des trois langues utilisées admirablement par le maître d'hôtel en expliquant les ingrédients des plats et la manière de les préparer.

En sortant de son premier cours, mon ami m'a cité les prénoms de quelques étudiantes ${ }^{4}$. Il voulait signaler discrètement leurs différences. Aussi a-t-il relevé un contraste dans leur manière de s'habiller, et une diversité dans leurs manières de voir les choses et d'analyser les comportements. À ses yeux, notre pays était comme prédestiné à la traduction. C'est un exercice quotidien, me disait-il. Les gens d'ici passent d'une langue à l'autre ou mélangent plusieurs langues. Frappé par ce qu'il avait

Meta, L, 1, 2005 
vu et entendu, il s'exclamait: «Quel paradis pour une formation en traduction!» Mais mon ami, tel que je le connais, ne voulait pas seulement faire l'éloge de mon pays favorable à l'enseignement de la traduction et l'interprétation, il voulait surtout savoir comment peut-on prétendre, cinq ans durant ${ }^{5}$, former des traducteurs ou des interprètes déjà formés. Ou plus précisément, quelle est la réaction des gens vis-à-vis de toute formation en traduction ou en interprétation dans un milieu si facile!

Quatre points font l'objet de réponse à une question si pertinente - le premier: la consommation et la production; le deuxième: le ré-apprentissage et la formation; le troisième: le parasitisme et le professionnalisme; le quatrième: le savoir-faire et la réflexion.

\section{La consommation et la production}

Très tôt les petits Libanais sont en contact avec au moins une langue autre que l'arabe. En famille, la conversation se passe souvent dans un mélange d'arabe littéraire ou dialectal et de français ou d'anglais. À l'école, dès la maternelle, l'enseignement est bilingue $^{6}$. Les petites mains glissent bien de la droite à la gauche pour l'arabe et de la gauche à la droite pour les autres langues. Les cartables remplis de livres d'arabe, de français et d'anglais, de cahiers pour chaque langue ou matière pèsent lourd. Les matières scientifiques sont dispensées au choix dans l'une des trois langues.

Arrivés au baccalauréat, les jeunes apprennent l'histoire de la littérature arabe et française ou anglaise. Les questions philosophiques sont traitées dans au moins trois langues.

Bilingue ou trilingue, le bachelier fait son choix universitaire. Les universités exigent alors de lui un niveau linguistique convenable. Les tests d'aptitude dans les trois langues font paraître la nécessité d'une mise à niveau ou d'un perfectionnement linguistique. Il est à signaler que devant de simples textes médiatiques, les candidats se trouvent mal à l'aise. Ils n'ont pas l'habitude de ce registre de langue; ils ont fréquenté, appris, les textes classiques ou scolaires. La langue demeure pour eux une langue écrite, celle des grands écrivains, ou poètes, ou philosophes, ou autrement dit, la langue de «l'examen» aboutissant à des réponses ou à des dissertations types.

Le bac en poche, le jeune universitaire en dehors de quelques moules ou recettes toutes faites, souffre au moins de cinq faiblesses: la première, comprendre et ré-exprimer un texte, ou une situation orale; la seconde, prendre la parole en public; la troisième structurer sa pensée et organiser ses idées; la quatrième, avoir le courage de dépasser le maniement restreint de la langue en vue de saisir toutes ses dimensions et par conséquent d'aller plus loin; la cinquième: oser la créativité.

Des cours intensifs ${ }^{7}$ sont organisés en ce sens trois mois durant, avant d'entreprendre le programme proprement dit. Le matériel pédagogique utilisé vise surtout à redonner confiance à l'étudiant et à le faire sortir de lui-même. Il lui revient de laisser ses empreintes sur son expression, de ne plus être répétitif se nourrissant de boîtes congelées ou de conserves mais d'aller vers la naturelle et de s'adonner au vrai. Bref de passer de la consommation à la production.

Mais qu'est-ce que la production dans une langue? Être productif, c'est être capable de nouveaux produits; or, les nouveaux produits dans une langue ce sont des possibilités infinies de combinaisons. Il s'agit d'un emploi dynamique et créatif de la langue. Ce ne sont plus les quelques formules de politesse, ou d'introduction, ou de 
conclusion, ou de transition qui classifient les utilisateurs de la langue, mais ce sont les capacités de créativité. C'est cette nouvelle relation d'amour entre l'utilisateur et la langue, et c'est justement à partir de cet amour et par lui que la langue s'offre dans toute son intimité. En échange, il lui confie le plus profond de ses secrets et de ses pensées.

Dans la formation du traducteur, à Beyrouth, c'est surtout la formation d'un productif n'ayant rien à envier à un écrivain qu'il faut viser; c'est dire combien cette tâche est difficile dans un milieu où les consommateurs de deux langues s'improvisent traducteurs ${ }^{8}$ ou interprètes. Toute une société s'étonne devant cette formation. Un travail en profondeur sur les mentalités devrait accompagner cette formation car à Beyrouth «chaque idée habite une maison, chaque mot est une ostentation, où l'on décharge pensées et caravanes».

\section{Le ré-apprentissage et la formation}

Dans une situation pareille à la nôtre, il semble mieux adapté de parler de ré-apprentissage pour aboutir à une formation. Le ré-apprentissage est défini non comme apprendre de nouveau, mais comme changement de fond dans les trois attitudes «concevoir», «recevoir» et "évaluer».

La première attitude est celle du professeur, qui n'ayant plus à être terrorisé par un programme et un examen «officiels» se plaît à préparer un cours sur mesure à partir des besoins de ses étudiants. Son enseignement devient individualisé, il peut dresser pour chacun de ses étudiants un bilan spécifique à partir duquel il rythme son cours et harmonise son enseignement. Mais cette attitude exige du professeur non plus les mêmes exercices répétés chaque année mais des exercices «frais » sur commande. À partir de cette préparation d'exercices faite à l'intention de ces étudiants, chaque étudiant se sent concerné, visé, ou plutôt, respecté. Inutile de signaler que le professeur appelle ses étudiants par leur nom, et connaît leurs erreurs, leurs faiblesses, leurs efforts. Quant à ce travail «fait main» en face d'un autre fait «en série», il est plus rentable car la magie de l'apprentissage s'installe et efface alors les failles et les lacunes. Cependant, est-il si simple de changer l'attitude du professeur? Serait-il prêt à changer son approche? Pouvons-nous lui demander de préparer son cours à partir des besoins de chaque groupe? Il est toujours difficile, voire impossible, dans certaines disciplines, de le convaincre: il trouve que cela est peut-être valable par exemple dans l'enseignement ou la mise à niveau des langues, mais pas dans l'enseignement de sa matière, comme si une certaine hiérarchie, une certaine noblesse entre les matières enseignées donnaient à certains professeurs une notoriété ou, comme si elles entouraient leur tête d'une auréole.

Un enseignement universitaire ne devrait-il pas permettre à l'étudiant de se réconcilier avec quelques matières abandonnées ou boudées par lui "grâce» à un enseignement répétitif, en chaîne, souvent dispensé dans le scolaire? N’est-ce pas là, à l'université, ce haut lieu de la formation, qu'il devrait développer ses connaissances et acquérir une formation ou un complément de formation?

Perdu, déboussolé, laissé à lui-même, la nostalgie d'un cours dicté, polycopié, appris par cour le prend mais cette crise au début, ou même durant la formation universitaire, est bénéfique car la rupture avec le cordon ombilical est en train de s'opérer: bientôt il se mettra tout seul debout et fera ses premiers pas. Il se prendra 
en charge et découvrira ses capacités infinies. La formation universitaire, et non le diplôme universitaire, est le visa nécessaire et suffisant pour un professionnalisme qui devrait orienter le marché du travail et par conséquent rejeter les parasites et les fantaisistes.

\section{Le parasitisme et le professionnalisme}

La situation linguistique aidant, le parasitisme fait face au professionnalisme. L'exemple le plus frappant est celui de cette «décision officielle» dans la réforme des programmes du baccalauréat libanais qui a confié l'enseignement de la traduction aux professeurs de langues et de littérature.

Incapables de saisir ou d'appliquer les exercices du livre de traduction préparé par les soins du Centre National de la Recherche et de Développement Pédagogiques ${ }^{9}$ et la collaboration de spécialistes, le ministère de l'Éducation nationale s'est trouvé dans une situation difficile. La suspension provisoire de la matière à l'examen officiel $^{10}$ fut la seule issue. Personne n'a eu le génie de penser aux professionnels de la matière formés à l'exercice du métier et à la pédagogie de la traduction ${ }^{11}$.

L'erreur de base ne réside même pas dans la problématique «l'enseignement de la traduction et la traduction dans l'enseignement ${ }^{12}{ }^{\prime}$, elle se situe à un niveau plus profond, celui de la reconnaissance de la traduction comme matière de plein droit, comme discipline ayant son programme, sa progression, ses stratégies, ses théories, ses approches et par conséquent ses professionnels.

Sans vouloir porter atteinte à nos collègues professeurs de langues et de lettres,ou les traiter de parasites, nous voulons ré-insister sur un point majeur: les contraintes à tenir rigoureusement compte de toutes les propriétés d'un texte, y compris de sa composition, de son rythme, de son ton, et qui concourent à lui donner son sens, sont un passage obligé à la fois du professeur et du traducteur, mais seul le traducteur est le voyageur entre les deux rives, c'est lui, et lui seul qui assure l'aller et retour critique entre la source et la cible ${ }^{13}$.

Le professionnel est celui qui, sans prétendre ou vouloir s'approprier une discipline et ses méthodes, est capable de s'en servir dans le strict cadre de sa profession. La formation du traducteur qui couvre plusieurs disciplines et matières ne fait pas de lui un professionnel dans ces disciplines et matières ${ }^{14}$.

Les cours de droit et d'économie par exemple ne font pas de lui un juriste ou un économiste, mais lui assurent un bagage cognitif sans lequel sa formation sera incomplète, son professionnalisme manqué.

\section{Le savoir-faire et la réflexion}

Dans la formation académique d'un traducteur ou d'un interprète, l'accent n'est pas seulement mis sur des techniques ou des moyens aboutissant à un exercice valable. Ce volet pratique de la formation est accompagné d'un autre, celui de la réflexion sur l'opération traduisante en tant qu'acte de communication. La querelle entre praticiens et théoriciens ${ }^{15}$ est vaine. Un effort d'analyse et de réflexion est exigé en même temps que l'exercice pratique. L'opération traduisante est un va-et-vient entre la pratique et la théorie. Mais quand et comment introduire la théorie dans la formation? Il est évident que la pratique en général devance la théorie; en effet, l'homme s'est 
exprimé au début, ensuite les grammairiens et les linguistes ont analysé, théorisé, réglementé son expression. De même, en traduction, les traducteurs ont traduit, ensuite les linguistes, les philosophes, les grammairiens et les traductologues se sont penchés sur leur savoir-faire, mais parfois ce sont eux-mêmes, les traducteurs, qui ont décrit leurs difficultés, ou analysé leurs démarches, ou émis quelques réflexions sur l'opération traduisante elle-même.

Le retour à ces balbutiements traductologiques à travers l'histoire des traducteurs et de la traduction est un heureux choix pour mener conjointement la pratique et la théorie. Les écrits traductologiques à travers les siècles montrent bien le développement de la pensée traductologique. Des hommes de lettres arabes tels que $\mathrm{Jahiz}^{16}$, à l'époque abbasside ou Sulaiman Al Boustany, n'ont pas manqué de définir la traduction et de décrire l'opération traduisante. Ces textes anciens n'ont rien à envier aux textes traductologiques de nos jours sauf que les anciens, à l'instar du bourgeois gentilhomme dans son apprentissage de la prose et de la poésie, faisaient de la traductologie sans s'en apercevoir.

L'introduction de ce volet traductologique lié à l'histoire de la traduction dans l'enseignement se fait en deux temps: le premier intégré aux exercices pratiques de la traduction dans toutes les combinaisons ${ }^{17}$ et dans tous les domaines ${ }^{18}$. Le second séparé, en tant que réflexion de synthèse menant à des pistes de recherche et de réflexion.

Dès la première année, l'enseignement se passe en interaction entre pratique et théorie, permettant ainsi au futur traducteur d'être capable d'agir et de réfléchir sur son action. Chaque langue lui apporte son expérience et le mène à réaliser comment et où elle s'entrecroise avec d'autres expériences dans d'autres langues. Cette réflexion voudrait aboutir à une constatation de valeur: toutes les expériences sont les mêmes, toutes les expériences sont différentes. N'est-ce pas là l'ultime position du traducteur?

La part de la réflexion dans l'enseignement/apprentissage de la traduction et de l'interprétation ne devrait pas se faire aux dépens de la pratique, l'inverse étant aussi valable; le dosage entre la réflexion et la pratique est pareil au jeu d'équilibre sans cesse assumé par le traducteur et l'interprète entre le texte source et le texte cible. En fin de compte, le traducteur et l'interprète ne seraient-ils pas des peseurs ${ }^{19}$ doublés d'équilibristes?

En accompagnant mon ami à l'aéroport, nous avons échangé ces quelques réflexions:

Enseigner la traduction et l'interprétation dans un milieu bilingue ou plurilingue, c'est travailler en terrain à la fois fécond et miné. Le premier objectif d'un enseignement dans cette situation dangereuse réside dans un déminage faisant souvent la cible de tireurs embusqués travaillant à la solde du parasitisme.

Il revient à la qualité de l'enseignement, à sa rigueur et à son dynamisme de rentabiliser la fécondité de ce terrain et par conséquent d'assurer une formation solide aux futurs traducteurs/interprètes.

Cependant, pouvons-nous parler de la qualité de la formation dans un foisonnement accru $^{20}$ d'écoles ou de centres d'enseignement de traduction et d'interprétation?

La qualification de toutes ces institutions dites de formation de traducteurs ou d'interprètes ne devrait-elle pas être à la charge de fédérations ou de conférences internationales ${ }^{21}$ ? Ne seraient-elles pas les instances adéquates pour accréditer cette formation? 
La formation continue ${ }^{22}$ ne devrait-elle pas accompagner le métier de traducteur et d'interprètes?

Avant de me quitter pour la zone consacrée aux voyageurs, mon ami m’a glissé ces quelques mots: «La qualité de l'accueil à Beyrouth ne réside pas seulement dans une hospitalité qui tient dans toute la magie de l'Orient ou dans quelques aspects folkloriques, mais elle réside surtout dans la manière d'être conscient des grands problèmes de la traduction et de l'interprétation et de vouloir participer avec ferveur à les résoudre ${ }^{23}$.»

\section{NOTES}

1. L'ÉTIB compte parmi son corps professoral quelques professeurs invités. Signalons à titre d'exemple: Mme Françoise De Dax D’Axat, Mme Hannelore Lee-Jahnke, M. Jean-René Ladmiral, M. Maurice Pergnier, M. Christian Balliu, M. Chawki Rayes, M. André Clas.

2. Le Liban a toujours été bilingue ou plurilingue, actuellement l'arabe est sa langue officielle, le français sa langue seconde, l'anglais, l'espagnol, l'italien, l'allemand ses langues étrangères. Notons la langue arménienne due à la présence des Arméniens à la suite du génocide.

3. Le mezzé: variété de petits plats accompagnés d'arak.

4. Les prénoms des Libanais sont occidentaux ou arabes: Mustapha ou Nour s'entourent de JeanClaude ou de Marie-Thérèse.

5. La formation à l'ÉTIB exige cinq ans répartis comme suit: $\mathrm{Bac}+3 \rightarrow$ licence en langues vivantes option traduction, puis 2 ans de traduction ou d'interprétation: $\mathrm{Bac}+3+2$. Le diplômé peut après trois ans d'expérience et, après l'étude de son dossier, s'inscrire au programme de doctorat.

6. Deux instituteurs assurent les cours en arabe et en français, un troisième, dès la classe de $6^{e}$ les rejoint pour l'anglais.

7. À l'ÉTIB, les trois premiers mois de la première année sont consacrés entièrement à la mise à niveau dans les trois langues: arabe/français/anglais à raison de 2 heures pour chaque langue sur un module de 100 heures.

8. En connaissant l'arabe et le français, les gens se croient traducteurs ou, interprètes et ne voient pas l'utilité de la formation.

9. Le premier livre en classe de première est intitulé Traduire: comprendre et faire comprendre, CNRDP, Sin - El - Fil, Liban, 1999. Le second en Terminal intitulé: «Traduire: interaction et créativité» CNRDP, Sin - El - Fil, Liban, 2000. Les deux livres sont accompagnés de deux livres du maître.

10. La suspension a été décidée en 2000, elle l'est toujours.

11. À l'ÉTIB, la pédagogie de l'enseignement de traduction figure dans le programme de la cinquième année.

12. Enseignement de la traduction et traduction dans l'enseignement, sous la direction de Jean Delisle et Hannelore Lee-Jahnke, préface de Maurice Pergnier, Les presses de l’Université d'Ottawa, Canada, 19998.

13. Jean-François Billeter, Leçon sur Tchouang - Tsen, édition ALLIA, Paris, 2002, p. 11.

14. Toutes ces matières sont connues à l'ETIB sous le nom de multidisciplinaires. Ils accompagnent l'étudiant durant ses trois premières années et sont dispensées dans l'une des trois langues de l'école.

15. Jean-René Ladmiral Traduire: théorèmes pour la traduction, PBP, Payot, Paris, 1979.

16. * AL JAHEZ, Kitab Al Hayawan, édition AL HILAL, Beyrouth, 1992.

* Sulaiman Al Boustany, l'Iliade d'Homère, traduction en arabe, Beyrouth, 1994.

* Claude-Gaspar Bachet De Meziriac, De la traduction, Introduction Michel Ballard, Artois Presses Université Presses de l'Université d'Ottawa, France 1998.

17. Les combinaisons à l'ETIB: $\mathrm{A}$ arabe, $\mathrm{B}$ français, $\mathrm{C}$ anglais: $\mathrm{A}-\mathrm{B} / \mathrm{A}-\mathrm{C} / \mathrm{B}-\mathrm{A} / \mathrm{B}-\mathrm{C} / \mathrm{C}-\mathrm{A} / \mathrm{C}-\mathrm{B}$.

18. Les domaines sont: le domaine juridique, le domaine économique, le domaine littéraire, le domaine médiatique, le domaine médical, le domaine technique.

19. Valery Larbaud, Sous l'invocation de Saint-Jérôme, Gallimard, coll. «Tel», Paris, 1997, p. 76.

20. Dix ans après la fondation de l'ETIB en 1980, plusieurs centres ou sections ou instituts académiques ont vu le jour s'inspirant largement de ses programmes.

21. Nous pensons notamment à la Fédération Internationale des Traducteurs (FIT) et à la Conférence Internationale (CIUTI) ainsi qu'à l'Association Internationale des Interprètes de Conférence (AIIC). 
22. Des sessions de formation continue sont organisées par la section de la formation continue à l'ÉTIB et la Commission de formation et de qualification des traducteurs à la FIT.

23. Trois publications de l'ETIB:

La collection SOURCE CIBLE éditée par l'ÉTIB, distribuée par la Librairie du Liban et dirigée par Henri Awaiss et Jarjoura Hardane cinq parutions jusqu'à présent:

Traduction: Approches et théories, Beyrouth, 1999.

Les langues à travers le SGAV, Beyrouth, 2001.

Terminologie de la traduction مصطلحات تعليم التزجمة, Beyrouth, 2002.

La traduction en partage, Beyrouth, 2002.

Du pareil au même, l'auteur face à son traducteur, Beyrouth 2003.

Les annales de l'Institut de Langues et de Traduction (ILT).

Terminus, bulletin du Centre d'études et de recherches en terminologie arabe (CERTA). 